University of Nebraska - Lincoln

DigitalCommons@University of Nebraska - Lincoln

3-1995

\title{
Energy and Angular Distributions of Electrons from Ion Impact on Atomic and Molecular Hydrogen. I. 20- 114-keV H$+\mathrm{H}_{2}$
}

\author{
M. W. Gealy \\ University of Nebraska - Lincoln \\ G. W. Kerby, III \\ University of Nebraska - Lincoln \\ Y.-Y. Hsu \\ University of Nebraska - Lincoln \\ M. Eugene Rudd \\ University of Nebraska - Lincoln, erudd@unl.edu
}

Follow this and additional works at: https://digitalcommons.unl.edu/physicsrudd

Part of the Physics Commons

Gealy, M. W.; Kerby, III, G. W.; Hsu, Y.-Y.; and Rudd, M. Eugene, "Energy and Angular Distributions of Electrons from Ion Impact on Atomic and Molecular Hydrogen. I. 20- 114-keV H+ $+\mathrm{H}_{2}$ " (1995). M. Eugene Rudd Publications. 33.

https://digitalcommons.unl.edu/physicsrudd/33

This Article is brought to you for free and open access by the Research Papers in Physics and Astronomy at DigitalCommons@University of Nebraska - Lincoln. It has been accepted for inclusion in M. Eugene Rudd Publications by an authorized administrator of DigitalCommons@University of Nebraska - Lincoln. 


\title{
Energy and angular distributions of electrons from ion impact on atomic and molecular hydrogen. I. 20-114-keV H${ }^{+}+\mathrm{H}_{2}$
}

\author{
M. W. Gealy, ${ }^{*}$ G. W. Kerby III, Y.-Y. Hsu, and M. E. Rudd \\ Department of Physics and Astronomy, University of Nebraska, Lincoln, Nebraska 68588-0111
}

(Received 30 August 1994; revised manuscript received 16 November 1994)

\begin{abstract}
Apparatus and procedures are described for the measurement of absolute cross sections, differential in ejected electron energy and angle, for ionization of atomic and molecular hydrogen by ion impact. A hemispherical electrostatic energy analyzer, rotatable from $15^{\circ}$ to $165^{\circ}$ with respect to the direction of the incident ion beam, was used to measure energy spectra of secondary electrons from 1.5 to $300 \mathrm{eV}$. Cross sections at ten angles (nine at some energies) and five incident-ion energies from 20 to $114 \mathrm{keV}$ for $\mathbf{H}^{+}+\mathbf{H}_{2}$ collisions are given. The doubly differential cross sections were integrated over angle and electron energy to obtain singly differential and total-ionization cross sections. The uncertainty in the doubly differential cross sections is $21 \%$ at a secondary energy of $1.5 \mathrm{eV}$ decreasing to $18 \%$ at $10 \mathrm{eV}$ and above. The total cross sections have a rms deviation of $12 \%$ from recommended values. A broad peak at $6 \mathrm{eV}$ in the energy spectrum of electrons from low-energy $\mathrm{H}^{+}+\mathrm{H}_{2}$ collisions is attributed to autoionization.
\end{abstract}

PACS number(s): 34.50.Fa

\section{INTRODUCTION}

Ionization is an important process in the deposition of energy of fast charged particles in matter. In ion collisions above about $100 \mathrm{keV} / \mathrm{u}$, ionization is not only the most probable of the elementary processes, but it also involves the greatest energy transfer per collision. Examples of fields in which data on collisional ionization are needed include thermonuclear and plasma studies, auroral and other upper atmospheric investigations, and studies of radiation effects in biological and other materials.

Since the final state of an ionizing collision involves the interaction of at least three bodies, calculations of ionization cross sections always require approximations. To assess the accuracy of proposed approximation methods, experimental cross sections are needed. Detailed experimental data, such as the doubly differential cross sections (DDCSs) reported here and the singly differential cross sections (SDCSs) obtained from them by integrating over angle have not only provided sensitive tests of theoretical descriptions of ionization, but have also brought to light previously unsuspected mechanisms for this process [1]. This investigation provides a data base of this type for the simplest possible target, the hydrogen atom. Because of the difficulty in producing a suitable atomic hydrogen target for such a measurement, this type of experiment has only recently been undertaken for electron impact [2] and has not previously been reported for ion impact.

Current methods of producing atomic hydrogen yield targets which also contain an appreciable fraction of molecular hydrogen. It is still possible to obtain cross

\footnotetext{
*Present address: Concordia College, Moorhead, MN 56562.
}

sections for $\mathrm{H}$ if the corresponding $\mathrm{H}_{2}$ cross sections are available. Since previously published DDCS data for $\mathbf{H}_{2}$ in this energy range were limited in accuracy and extent, these cross sections were also measured as part of this investigation. Blauth's early measurements [3] were confined to a single angle. The data of Kuyatt and Jorgensen [4] at 50-100 keV were inaccurate because the low efficiency of their system for low-energy electrons resulted in an incorrect normalization. The single measurement at $50 \mathrm{keV}$ of Gibson and Reid [5] covered angles down to $0^{\circ}$ but only up to $100^{\circ}$ and therefore gives little information about ejection of electrons into the backward hemisphere. Furthermore, the accuracy of their angular distributions was brought into question by Cheng, Rudd, and Hsu [6]. Rudd and Jorgensen [7] presented data only at $100 \mathrm{keV}$. DDCSs measured by Rudd, Sautter, and Bailey [8] at 100-300 keV and by Rudd [9] at 5-100 keV were evidently too large since their integrals were $35-100 \%$ higher than the total-ionization cross sections (TICSs) measured by more accurate direct methods $[10,11]$. Toburen and Wilson's DDCS data [12] at $300-1500 \mathrm{keV}$ are above the energy range of the present work. The new apparatus used in this investigation was designed to reduce the errors in measuring low-energy electrons inherent in earlier measurements from this laboratory. Therefore, these data for $\mathrm{H}_{2}$ targets represent an important addition to the data base in this projectileenergy region.

In this paper-paper I of a series of four-the apparatus and experimental methods are described for determining DDCSs for the collision of ions with $\mathrm{H}_{2}$ and for extracting cross sections for $\mathrm{H}$ from measurements on mixed $\mathbf{H}$ and $\mathrm{H}_{2}$ targets. The results of the $\mathbf{H}^{+}+\mathrm{H}_{2}$ measurements are presented here. Paper II [13] describes the $\mathrm{H}^{+}+\mathbf{H}$ data and also presents new theoretical calculations on that system. Similar results for $\mathrm{He}^{+}+\mathrm{H}_{2}$ and $\mathrm{He}^{+}+\mathrm{H}$ are planned to be given in papers III and IV. 


\section{APPARATUS}

\section{A. Primary-ion beam}

Ions are extracted from a radio frequency ( $\mathrm{rf}$ ) ion source, accelerated, magnetically analyzed, and electrostatically focused before entering the interaction region. The 1.4-m-long region between the analyzing magnet and the stainless-steel collision chamber is separately pumped to minimize contamination of the beam by charge transfer with the residual gas. The shielded beam defining apertures are biased at $+67.5 \mathrm{~V}$ to prevent secondary electrons from entering the collision region.

The ions are collected in a coaxial double Faraday cup and the current integrated for the duration of the electron counting. The outer cup is $0.95 \mathrm{~cm}$ in diameter and has a $0.32-\mathrm{cm}$ hole which allows the central part of the beam to strike the inner "cup," an electrode directly behind the hole. The inner and outer cups were biased at +67.5 and $+45 \mathrm{~V}$, respectively, to prevent the escape of secondary electrons. A grounded shield surrounds the cups. Although during data taking the currents from both cups were combined, they could be read separately to provide coarse monitoring of the beam profile. At energies of $50 \mathrm{keV}$ and above, the inner cup typically received over $90 \%$ of the beam. At lower energies the fraction ranged from 70-90\%, depending on the focusing. The maximum angular divergence of the beam was $\pm 0.24^{\circ}$ and its maximum diameter at the collision center was $2.4 \mathrm{~mm}$.

\section{B. Atomic hydrogen target source}

The atomic hydrogen target is obtained by dissociating $\mathrm{H}_{2}$ in a rf discharge source of the type described by Slevin and Stirling [14], obtained commercially [15]. Mounted on the top of the collision chamber, the source directs the target beam downward across the ion beam and directly into a diffusion pump with a pumping speed of $17001 / \mathrm{s}$ for hydrogen. The projectile beam intersects the target beam approximately $4 \mathrm{~mm}$ beneath the nozzle. With a frequency of $35 \mathrm{MHz}$, a power of about $4 \mathrm{~W}$, and a source pressure of $0.3 \mathrm{mB}$, the dissociation fraction $D$ varied from $55 \%$ to $82 \%$ over a period of several months during which the final data were taken. The average value was $74 \%$ and the density of $\mathrm{H}$-atom targets in the interaction region was approximately $5 \times 10^{11} \mathrm{~cm}^{-3}$. Since $D$ varied slightly from day to day and from angle to angle, it was measured separately for each run. The change over the time of a run was negligible.

A rf coil surrounds the water-cooled Pyrex cavity in which the discharge is maintained. Since cooling the walls of the discharge tube reduces recombination, a refrigerator in the recirculating system was provided which kept the temperature at $10^{\circ} \mathrm{C}$. Hydrogen effuses through a 1-mm-diam capillary tube into the collision chamber. There is an S-shaped kink in the capillary near the exit nozzle to trap most of the ultraviolet radiation. Contrary to expectations, we found that greater dissociation was obtained when hydrogen was admitted to the source through a mechanical leak than through the palladium leak supplied by the manufacturer. Hydrogen at
99.999\% purity provided no improvement over $99.99 \%$ hydrogen. An all-metal gas transport system was used.

As supplied by the manufacturer, electrical shielding surrounded the rf coil but not the glass nozzle. Since it is important that there be no unshielded insulating material near the interaction region, an additional grounded copper shield was built which covers the nozzle except for about $1 \mathrm{~mm}$ of the very tip, which was painted with colloidal graphite and electrically grounded. All other insulating material and electrical cables in the chamber are also well shielded.

Because of concern that in spite of the shielding, fields from the rf coil could affect the trajectories of the ejected electrons, an electrical switching system was added. This switches the rf power at a frequency of $50 \mathrm{~Hz}$ with a $50 \%$ duty cycle and simultaneously switches the counter gate so that electrons are recorded only during the part of the cycle when the rf power is off. It was found that a satisfactory degree of dissociation could be maintained when delivering the $\mathrm{rf}$ power to the discharge in the ac mode. Measurements showed that the dissociation fraction of the gas from the source decayed with a time constant of about $0.2 \mathrm{~s}$ after the power was shut off; thus the change over a half period of the chopping signal was small. Even though results obtained with and without the switching system were found to be essentially the same even at count rates as low as one count per hundred seconds, all final data were taken using the ac mode.

To show that the target beam from a similar rf discharge source was free of contamination by metastable $\mathbf{H}(2 S)$ atoms, Houver, Fayeton, and Barat [16] measured the projectile energy-loss spectrum in collisions of protons with the source gas and found no evidence of atomic targets existing in the $n=2$ level. The forward power to their source was approximately ten times that used in the present work and their frequency was $120 \mathrm{Mhz}$. While we did not have as sensitive a method for detecting metastable atoms, we measured the electron energy spectrum from 50 to $200 \mathrm{eV}$ at an emission angle of $30^{\circ}$ in collisions of $70-\mathrm{keV}$ protons with the rf-power-on target gas. All three of the theoretical calculations given by Schultz et al. [17] for a $\mathbf{H}(2 S)$ target predict a prominent binary-encounter peak centered at an electron energy of approximately $100 \mathrm{eV}$, which is nearly four times as large as that for a ground-state target. Based on our failure to detect such a peak we estimate that our target gas contained no more than $0.5 \%$ of metastable atomic hydrogen.

\section{Electron energy analysis and detection}

The interaction volume is defined by two grounded rectangular apertures of widths 4 and $3 \mathrm{~mm}$ which collimate the electrons entering the electrostatic analyzer. These apertures define an effective solid-angle path-length integral over the observed interaction volume to be $(l \Delta \Omega)_{\mathrm{eff}}=\int \Omega(z) d z=1.2 \times 10^{-3} \mathrm{~cm} \mathrm{sr} / \sin \theta$, where $z$ is the distance along the projectile beam and $\theta$ the angle between the electron trajectory and the beam. The full width at half maximum (FWHM) of the angular acceptance of the collimating system is $\pm 4.6^{\circ}$. The analyzer is 
continuously rotatable from $15^{\circ}$ to $100^{\circ}$ on one side of the projectile beam and from $80^{\circ}$ to $165^{\circ}$ on the other side. Measurements at $90^{\circ}$ on both sides of the beam indicated no appreciable asymmetry.

Electrons that pass through the collimator enter the hemispherical electrostatic energy analyzer made of oxygen-free high-conductivity copper with inner and outer radii of 2.5 and $3.5 \mathrm{~cm}$, respectively. The surfaces exposed to electrons are coated with carbon soot to reduce reflected and secondary electrons [18]. The analyzer has a resolution FWHM of $5.0 \%$. Since the entrance and exit apertures may be biased with a voltage $V_{a}$ to preaccelerate the particles entering the analyzer, the resolution is $\Delta W=0.050\left(W+e V_{a}\right)$, where $W$ is the kinetic energy of the secondary electrons and $e$ is the magnitude of the electron charge. $V_{a}$ was kept at a small positive potential (usually $5 \mathrm{~V}$ ) to improve collection efficiency for low-energy electrons.

Electrons leaving the analyzer were detected by a channel electron multiplier (CEM) the cone of which was biased at $+100 \mathrm{~V}$. Earlier measurements in this laboratory [19] showed that with this bias the CEM efficiency is constant within $4 \%$ over the energy range of this experiment. Pulse-height distribution measurements showed that at the discriminator setting used the discriminator efficiency was $99 \%$. In our energy region the CEM detector efficiency $[19,20]$ was $96 \%$, thus giving an overall detection efficiency $\epsilon$ of $95 \%$ for electrons. Since the efficiency decreases with increasing count rate, proton beam currents were adjusted to keep the count rate below about $2500 \mathrm{~s}^{-1}$, where the drop in the efficiency is negligible.

Magnetic shielding plus a single coil outside the chamber kept the magnetic field below $5 \mathrm{mG}$ near the collision center and under $10 \mathrm{mG}$ elsewhere in the chamber. The entire collimator, analyzer, and detector system was enclosed in a grounded copper housing for electrostatic shielding.

\section{EXPERIMENTAL METHOD}

Because the $\mathbf{H}$-atom source produces a mixture of $\mathbf{H}$ and $\mathrm{H}_{2}$, the determination of absolute cross sections for atomic targets involves four steps: (i) the dissociation fraction is measured, (ii) electron counts were taken with the rf power on to obtain the ratio $R_{12}$ of the $\mathrm{H}$ to $\mathrm{H}_{2}$ cross sections and with the rf power off to obtain the relative values of the $\mathrm{H}_{2}$ cross sections, (iii) using a static gas absolute measurements are made of the cross sections for $\mathrm{H}_{2}$ targets to normalize the relative data, and (iv) the cross sections for $\mathbf{H}$ are calculated from the ratio measurements and the normalized $\mathrm{H}_{2}$ data.

\section{A. Measurement of the dissociation fraction $D$}

The quantity $D$, the fraction of $\mathrm{H}_{2}$ molecules dissociated, was measured by a method which takes advantage of the fact that the energy spectrum of positive ions from $\mathrm{H}^{+}+\mathrm{H}_{2}$ collisions has a broad peak centered near $9 \mathrm{eV}$ resulting from the dissociation of the $2 p \sigma_{u}$ and other nearby states of $\mathrm{H}_{2}{ }^{+}$[21]. These states decay with about
$18 \mathrm{eV}$ of energy, which is shared by the two fragments. Double ionization of $\mathrm{H}_{2}$ also contributes to the 9-eV peak [22]. The angular distribution of the $9-\mathrm{eV}$ ions is nearly isotropic [23] over our range of energies, but this is not essential to the method. Since most of the recoil ions from $\mathrm{H}^{+}+\mathbf{H}$ collisions are confined to energies well below $1 \mathrm{eV}$, the ion signal at $9 \mathrm{eV}$ is proportional to the density of $\mathbf{H}_{2}$. The dissociation fraction is then

$$
D=1-\frac{S_{i}^{\text {on }}}{S_{i}^{\text {off }}},
$$

where $S_{i}^{\text {on }}$ and $S_{i}^{\text {off }}$ are the 9-eV ion signals with the $\mathrm{rf}$ power on and off, respectively. The ions are measured with the same collimation system and electrostatic analyzer used for electrons, but with the polarities of the electrode potentials reversed and the cone of the CEM biased at $-2100 \mathrm{~V}$.

Shyn [2] measured dissociation fractions with a quadrupole mass spectrometer. Our method has the advantage that the dissociation and the electron signals are measured with the same detector viewing precisely the same interaction region. The size of the interaction region varies with scattering angle $\theta$ and there is some spatial variation in $D$. But in this method the ratios of signals with the rf power on and off are still equal to the corresponding ratios of density path-length integrals and are therefore independent of the collision product being detected. Furthermore, since $D$ is obtained from ratios of ion signals and the cross sections are obtained from ratios of electron signals, the measurements of $D$ and $R_{12}$ are independent of detector efficiency.

As a check on this method of measuring $D$, slow ions formed in collisions of $\mathrm{H}^{+}$with the rf source on and off were drawn out of the interaction region by a weak electric field and analyzed with an auxiliary low-resolution magnetic mass spectrometer installed only for this diagnostic measurement. Effective target densities were calculated from these ion signals and known cross sections for formation of slow ions [24,25]. Assuming a uniform temperature for all target particles in both "on" and "off" rf source conditions allows us to solve for $D$ using any two of the three densities $n_{2}^{\text {off }}, n_{2}^{\text {on }}$, and $n_{1}^{\text {on }}$, where 1 and 2 refer to $\mathrm{H}$ and $\mathrm{H}_{2}$, respectively. In a typical measurement, $D$ values of $0.689,0.691$, and 0.684 were obtained from the three combinations. The self-consistency of these results indicates that the assumption of a uniform temperature is appropriate. Although some $\mathrm{H}^{+}$ions are produced from $\mathbf{H}_{2}$ targets by dissociative ionization, these ions have several eV of kinetic energy, as mentioned above. Since they are isotropically distributed and the extracting field was weak, a negligible fraction of such ions were collected in this measurement. Dissociation fractions measured at the same time over slightly different interaction volumes by the magnetic method and the 9-eV-ion method were in approximate agreement, giving us confidence in the accuracy of the latter method.

\section{B. Measurement of $\mathbf{H}$-to- $\mathrm{H}_{2}$ cross-section ratios}

In the crossed-beam configuration used in this part of the measurement the background pressure in the scatter- 
TABLE I. Measured values of $\sigma_{2}(W, \theta)$ in units of $10^{-20} \mathrm{~cm}^{2} / \mathrm{eV} \mathrm{sr}, \sigma_{2}(W)$ in units of $10^{-20} \mathrm{~cm}^{2} / \mathrm{eV}, \sigma_{2}(\theta)$ in units of $10^{-20}$ $\mathrm{cm}^{2} / \mathrm{sr}$, and $\sigma_{i}$ (lower right-hand corner) in units of $10^{-20} \mathrm{~cm}^{2}$ for secondary-electron production in $20-\mathrm{keV} \mathrm{H}^{+}+\mathrm{H}_{2}$ collisions. Numbers in brackets are powers of 10 by which quantities are to be multiplied.

\begin{tabular}{|c|c|c|c|c|c|c|c|c|c|c|}
\hline$W(\mathrm{eV})$ & $15^{\circ}$ & $20^{\circ}$ & $30^{\circ}$ & $50^{\circ}$ & $70^{\circ}$ & $90^{\circ}$ & $110^{\circ}$ & $130^{\circ}$ & $160^{\circ}$ & $\sigma(\boldsymbol{W})$ \\
\hline 1.5 & 652 & 481 & 276 & 90.3 & 44.4 & 27.9 & 18.5 & 18.5 & 20.9 & 989 \\
\hline 2 & 677 & 503 & 247 & 78.5 & 38.7 & 24.9 & 16.6 & 15.9 & 16.7 & 913 \\
\hline 3 & 681 & 470 & 202 & 64.8 & 35.3 & 21.7 & 15.1 & 14.4 & 13.0 & 828 \\
\hline 5 & 570 & 333 & 135 & 51.0 & 28.5 & 19.2 & 14.1 & 13.9 & 14.0 & 680 \\
\hline 7.5 & 302 & 187 & 100 & 44.1 & 23.5 & 15.4 & 11.6 & 11.4 & 11.1 & 468 \\
\hline 10 & 187 & 139 & 88.2 & 37.9 & 18.8 & 11.6 & 8.07 & 6.87 & 6.33 & 344 \\
\hline 15 & 137 & 111 & 69.6 & 25.1 & 10.6 & 6.13 & 3.51 & 2.81 & 1.88 & 225 \\
\hline 20 & 93.1 & 75.2 & 46.2 & 14.5 & 5.92 & 3.24 & 1.90 & 1.53 & 1.06 & 141 \\
\hline 30 & 37.4 & 30.9 & 18.6 & 5.09 & 2.04 & 1.17 & 0.711 & 0.713 & 0.323 & 54.1 \\
\hline 50 & 4.60 & 3.82 & 2.12 & 0.565 & 0.263 & 0.159 & 0.112 & 0.153 & 0.0477 & 6.62 \\
\hline 75 & 0.290 & 0.264 & 0.124 & 0.0406 & 0.0228 & & 0.0193 & & & 0.390 \\
\hline 100 & 0.0495 & 0.0725 & & $9.81[-3]$ & & & & & & 0.0269 \\
\hline 130 & 0.0259 & & & & & & & & & \\
\hline$\sigma(\theta)$ & 7000 & 4970 & 2750 & 967 & 472 & 291 & 197 & 187 & 174 & 10300 \\
\hline
\end{tabular}

ing chamber was typically less than $10^{-5}$ Torr and that in the differentially pumped beam line about $10^{-6}$ Torr. The effective pressure at the center of the interaction volume was several times $10^{-5}$ Torr. For each run counts were recorded for electron energies from 1.5 to $300 \mathrm{eV}$ (in a few cases to $400 \mathrm{eV}$ ) or to an energy at which the count rate was too low to yield satisfactory statistics.

The ratio $R_{12}$ of atomic to molecular cross sections is given by

$$
R_{12}=\frac{\sigma_{1}(W, \theta)}{\sigma_{2}(W, \theta)}=\frac{1}{\sqrt{2} D}\left[\frac{S_{e}^{\text {on }}}{S_{e}^{\text {off }}}-1+D\right]
$$

where $S_{e}^{\text {on }}$ and $S_{e}^{\text {off }}$ are the background-corrected electron signals with the rf on and off, respectively. This is the equation stated by Shyn [2] and is equivalent to the one derived by Brackmann and Fite [26] for a different experimental configuration. The derivation of this equation assumes that (i) the effective temperatures of $\mathrm{H}$ and $\mathrm{H}_{2}$ are the same and that they do not change when the rf power is switched on and (ii) the spatial distributions of $\mathrm{H}$ and $\mathrm{H}_{2}$ target particles are the same in the dissociation measurement as for the electron measurement. Assumption (i) was discussed in Sec. III A. Assumption (ii) is satisfied in our method of measuring $D$ since the same analyzer system is used for ions as for electrons.

TABLE II. Same as Table I, but for $48 \mathrm{keV}$.

\begin{tabular}{|c|c|c|c|c|c|c|c|c|c|c|c|}
\hline$W(\mathrm{eV})$ & $15^{\circ}$ & $20^{\circ}$ & $30^{\circ}$ & $50^{\circ}$ & $70^{\circ}$ & $90^{\circ}$ & $110^{\circ}$ & $130^{\circ}$ & $150^{\circ}$ & $165^{\circ}$ & $\sigma(W)$ \\
\hline 1.5 & 830 & 704 & 488 & 196 & 79.2 & 39.9 & 23.2 & 20.9 & 23.4 & 22.2 & 1570 \\
\hline 2 & 794 & 686 & 466 & 182 & 72.5 & 35.5 & 20.9 & 18.0 & 19.8 & 19.5 & 1460 \\
\hline 3 & 774 & 635 & 416 & 150 & 59.4 & 28.2 & 17.3 & 14.2 & 16.3 & 16.6 & 1290 \\
\hline 5 & 709 & 561 & 340 & 113 & 43.3 & 19.8 & 12.5 & 11.3 & 12.3 & 12.2 & 1050 \\
\hline 7.5 & 634 & 473 & 258 & 90.9 & 32.8 & 14.1 & 9.86 & 8.76 & 9.33 & 9.52 & 856 \\
\hline 10 & 565 & 393 & 205 & 73.0 & 25.7 & 10.4 & 7.16 & 5.99 & 5.89 & 5.83 & 708 \\
\hline 15 & 422 & 262 & 143 & 54.4 & 16.9 & 6.19 & 3.85 & 2.64 & 2.07 & 1.51 & 510 \\
\hline 20 & 283 & 183 & 108 & 39.8 & 10.7 & 3.33 & 2.06 & 1.36 & 1.14 & 0.760 & 353 \\
\hline 30 & 130 & 96.0 & 64.1 & 21.6 & 4.62 & 1.50 & 0.900 & 0.590 & 0.497 & 0.284 & 179 \\
\hline 50 & 35.8 & 35.3 & 24.3 & 5.51 & 0.976 & 0.387 & 0.244 & 0.158 & 0.122 & & 52.3 \\
\hline 75 & 11.6 & 11.1 & 6.43 & 0.977 & 0.189 & 0.0903 & 0.0632 & 0.0442 & 0.0286 & & 13.7 \\
\hline 100 & 3.16 & 2.74 & 1.32 & 0.166 & 0.0408 & 0.0206 & 0.0152 & 0.0121 & $4.18[-3]$ & & 3.12 \\
\hline 130 & 0.485 & 0.338 & 0.159 & 0.0208 & $6.19[-3]$ & & $2.24[-3]$ & & $6.65[-4]$ & & 0.439 \\
\hline 160 & 0.0533 & 0.0382 & 0.0174 & $2.30[-3]$ & & & & & & & 0.0459 \\
\hline 200 & & & $8.13[-4]$ & & & & & & & & \\
\hline$\sigma(\theta)$ & 15000 & 11300 & 6910 & 2380 & 784 & 338 & 211 & 175 & 181 & 166 & 21400 \\
\hline
\end{tabular}


TABLE III. Same as Table I, but for $67 \mathrm{keV}$.

\begin{tabular}{|c|c|c|c|c|c|c|c|c|c|c|}
\hline$W(\mathrm{eV})$ & $20^{\circ}$ & $30^{\circ}$ & $50^{\circ}$ & $70^{\circ}$ & $90^{\circ}$ & $110^{\circ}$ & $130^{\circ}$ & $150^{\circ}$ & $165^{\circ}$ & $\sigma(W)$ \\
\hline 1.5 & 652 & 466 & 223 & 98.2 & 48.9 & 31.4 & 24.0 & 25.4 & 24.7 & 1650 \\
\hline 2 & 613 & 441 & 208 & 88.9 & 42.8 & 26.4 & 20.2 & 22.1 & 21.2 & 1520 \\
\hline 3 & 573 & 395 & 181 & 74.7 & 34.7 & 21.0 & 16.6 & 18.0 & 18.4 & 1340 \\
\hline 5 & 496 & 324 & 143 & 58.2 & 24.6 & 14.7 & 12.4 & 12.8 & 13.0 & 1090 \\
\hline 7.5 & 422 & 267 & 112 & 44.3 & 17.5 & 10.4 & 8.90 & 9.39 & 9.63 & 876 \\
\hline 10 & 354 & 216 & 92.2 & 34.7 & 12.6 & 7.68 & 6.18 & 6.08 & 6.04 & 710 \\
\hline 15 & 268 & 152 & 67.1 & 23.7 & 7.25 & 3.87 & 2.58 & 2.01 & 1.47 & 504 \\
\hline 20 & 210 & 117 & 50.4 & 15.3 & 3.89 & 1.90 & 1.37 & 1.06 & 0.798 & 375 \\
\hline 30 & 121 & 72.1 & 30.7 & 7.10 & 1.58 & 0.865 & 0.545 & 0.434 & 0.251 & 216 \\
\hline 50 & 45.8 & 31.3 & 11.5 & 1.62 & 0.400 & 0.218 & 0.136 & 0.0980 & & 80.8 \\
\hline 75 & 19.6 & 13.0 & 3.08 & 0.325 & 0.107 & 0.0615 & 0.0368 & 0.0258 & & 29.9 \\
\hline 100 & 9.21 & 5.10 & 0.737 & 0.0839 & 0.035 & 0.0198 & 0.0115 & $7.39[-3]$ & & 12.0 \\
\hline 130 & 2.87 & 1.20 & 0.130 & 0.0198 & $9.42[-3]$ & $5.31[-3]$ & $3.45[-3]$ & $1.94[-3]$ & & 3.30 \\
\hline 160 & 0.637 & 0.225 & 0.0234 & & & $1.46[-3]$ & $8.53[-4]$ & $6.57[-4]$ & & 0.687 \\
\hline 200 & 0.0643 & 0.0230 & $4.27[-3]$ & & & $1.85[-4]$ & & $1.76[-4]$ & & 0.0731 \\
\hline 250 & $3.26[-3]$ & & $1.55[-3]$ & & & & & & & $5.51[-3]$ \\
\hline 300 & $1.23[-4]$ & & $1.06[-3]$ & & & & & & & $2.08[-3]$ \\
\hline$\sigma(\theta)$ & 12000 & 7540 & 3160 & 1060 & 409 & 247 & 190 & 188 & 178 & 23900 \\
\hline
\end{tabular}

\section{Measurement of cross sections for $\mathbf{H}_{2}$}

The rf-power-off electron signals $S_{e}^{\text {off }}$, measured for use in Eq. (2), could also be used to obtain relative values of the DDCSs for $\mathbf{H}_{2}$ and since these were already at hand, they needed only to be put on an absolute basis. To do this the flow of gas from the chamber to the diffusion pump was throttled to reduce the gas load on the backing pump and the collision chamber was flooded with $\mathrm{H}_{2}$ to a pressure of about $0.1 \mathrm{mTorr}$, thus forming an essentially static gas target. The density $n$ of target particles was calculated from the ideal-gas law using measurements of the chamber pressure by a capacitance manometer and assuming the gas to be at room temperature. Absolute DDCSs for each electron energy and scattering angle were determined from the equation

$$
\sigma_{2}(W, \theta)=\frac{N_{e}}{\Delta W \epsilon n N_{i}(l \Delta \Omega)_{\mathrm{eff}}},
$$

where $N_{e}$ is the number of electrons, corrected for absorption and for background, that were counted during

TABLE IV. Same as Table I, but for $95 \mathrm{keV}$.

\begin{tabular}{|c|c|c|c|c|c|c|c|c|c|c|c|}
\hline$W(\mathrm{eV})$ & $15^{\circ}$ & $20^{\circ}$ & $30^{\circ}$ & $50^{\circ}$ & $70^{\circ}$ & $90^{\circ}$ & $110^{\circ}$ & $130^{\circ}$ & $150^{\circ}$ & $165^{\circ}$ & $\sigma(W)$ \\
\hline 1.5 & 705 & 553 & 473 & 254 & 80.8 & 68.3 & 41.4 & 33.0 & 33.8 & 35.7 & 1760 \\
\hline 2 & 599 & 492 & 416 & 236 & 95.2 & 57.8 & 35.9 & 28.8 & 29.1 & 32.6 & 1600 \\
\hline 3 & 463 & 409 & 337 & 195 & 96.4 & 43.9 & 25.6 & 20.7 & 21.1 & 23.9 & 1300 \\
\hline 5 & 369 & 325 & 266 & 148 & 74.8 & 31.2 & 17.2 & 13.7 & 13.7 & 14.1 & 992 \\
\hline 7.5 & 309 & 263 & 210 & 115 & 56.8 & 21.7 & 11.6 & 9.43 & 9.27 & 9.13 & 770 \\
\hline 10 & 259 & 221 & 170 & 94.8 & 45.3 & 16.0 & 8.28 & 6.55 & 6.12 & 5.68 & 618 \\
\hline 15 & 208 & 167 & 128 & 71.0 & 31.9 & 9.10 & 4.12 & 2.89 & 2.29 & 2.07 & 451 \\
\hline 20 & 182 & 137 & 100 & 55.5 & 22.0 & 5.08 & 2.16 & 1.55 & 1.33 & 1.29 & 349 \\
\hline 30 & 131 & 90.5 & 63.7 & 35.1 & 11.8 & 2.03 & 0.934 & 0.659 & 0.573 & 0.626 & 221 \\
\hline 50 & 55.6 & 40.3 & 30.6 & 16.5 & 3.44 & 0.506 & 0.262 & 0.195 & 0.182 & 0.204 & 95.7 \\
\hline 75 & 20.5 & 17.5 & 15.1 & 6.80 & 0.817 & 0.143 & 0.0784 & 0.0642 & 0.0646 & 0.0711 & 38.8 \\
\hline 100 & 11.4 & 10.3 & 8.71 & 2.55 & 0.237 & 0.0536 & 0.0322 & 0.0241 & 0.0236 & 0.0259 & 19.0 \\
\hline 130 & 6.93 & 6.12 & 4.57 & 0.698 & 0.0648 & 0.0191 & 0.0108 & $8.39[-3]$ & $7.89[-3]$ & $8.71[-3]$ & 9.06 \\
\hline 160 & 3.92 & 3.44 & 1.83 & 0.183 & 0.0209 & $7.72[-3]$ & $3.73[-3]$ & $2.39[-3]$ & $2.63[-3]$ & $2.69[-3]$ & 3.88 \\
\hline 200 & 1.12 & 1.07 & 0.424 & 0.0316 & $4.34[-3]$ & $1.95[-3]$ & $8.84[-4]$ & & $5.10[-4]$ & $8.38[-4]$ & 0.929 \\
\hline 250 & 0.165 & 0.150 & 0.0516 & $4.73[-3]$ & & $7.05[-4]$ & & & & $2.54[-4]$ & 0.127 \\
\hline 300 & 0.0189 & 0.0181 & $6.57[-3]$ & $6.85[-4]$ & & & & & & & 0.0150 \\
\hline$\sigma(\theta)$ & 11500 & 9070 & 7070 & 3680 & 1330 & 537 & 292 & 229 & 224 & 229 & 24100 \\
\hline
\end{tabular}


TABLE V. Same as Table I, but for $114 \mathrm{keV}$.

\begin{tabular}{|c|c|c|c|c|c|c|c|c|c|c|c|}
\hline$W(\mathrm{eV})$ & $15^{\circ}$ & $20^{\circ}$ & $30^{\circ}$ & $50^{\circ}$ & $70^{\circ}$ & $90^{\circ}$ & $110^{\circ}$ & $130^{\circ}$ & $150^{\circ}$ & $165^{\circ}$ & $\sigma(W)$ \\
\hline 1.5 & 366 & 329 & 285 & 167 & 101 & 57.6 & 42.2 & 31.9 & 31.8 & 30.5 & 1260 \\
\hline 2 & 365 & 330 & 278 & 169 & 99.9 & 53.9 & 36.9 & 27.7 & 26.5 & 25.8 & 1220 \\
\hline 3 & 334 & 313 & 250 & 155 & 89.9 & 46.4 & 28.4 & 21.7 & 21.3 & 20.8 & 1080 \\
\hline 5 & 270 & 259 & 203 & 128 & 71.6 & 34.2 & 18.6 & 14.4 & 14.4 & 14.4 & 849 \\
\hline 7.5 & 228 & 209 & 166 & 101 & 55.7 & 24.6 & 12.4 & 9.77 & 9.82 & 9.83 & 668 \\
\hline 10 & 183 & 170 & 136 & 84.7 & 45.1 & 18.1 & 8.74 & 6.81 & 6.34 & 5.96 & 535 \\
\hline 15 & 142 & 125 & 99.0 & 63.7 & 32.7 & 10.7 & 4.18 & 2.88 & 2.17 & 1.71 & 381 \\
\hline 20 & 118 & 100 & 76.9 & 48.2 & 23.0 & 5.95 & 2.08 & 1.44 & 1.12 & 0.888 & 283 \\
\hline 30 & 74.1 & 66.0 & 47.7 & 31.6 & 12.9 & 2.39 & 0.821 & 0.534 & 0.414 & 0.287 & 170 \\
\hline 50 & 38.8 & 31.1 & 22.9 & 15.4 & 4.23 & 0.532 & 0.200 & 0.130 & 0.066 & 0.0572 & 78.5 \\
\hline 70 & 14.6 & 13.6 & 11.7 & 7.47 & 1.07 & 0.125 & 0.0538 & 0.0346 & 0.0231 & $5.20[-3]$ & 34.0 \\
\hline 100 & 7.45 & 7.81 & 7.33 & 3.57 & 0.281 & 0.0425 & 0.0199 & 0.0109 & $8.03[-3]$ & $2.59[-3]$ & 17.5 \\
\hline 130 & 4.80 & 5.03 & 4.62 & 1.23 & 0.0728 & 0.0142 & $7.90[-3]$ & $4.24[-3]$ & $2.96[-3]$ & $4.85[-4]$ & 8.99 \\
\hline 160 & 3.46 & 3.21 & 2.65 & 0.364 & 0.0217 & $6.11[-3]$ & $3.07[-3]$ & $1.60[-3]$ & $1.30[-3]$ & $4.21[-4]$ & 4.86 \\
\hline 200 & 1.97 & 1.67 & 0.882 & 0.0688 & $5.55[-3]$ & $1.99[-3]$ & $8.16[-4]$ & & $7.86[-4]$ & $9.92[-5]$ & 1.87 \\
\hline 250 & 0.530 & 0.413 & 0.146 & $8.68[-3]$ & $8.64[-4]$ & & & & & & 0.393 \\
\hline 300 & 0.0874 & 0.0609 & 0.0189 & $1.34[-3]$ & & & & & & & 0.0610 \\
\hline 400 & $1.56[-3]$ & $9.64[-4]$ & & & & & & & & & $7.15[-4]$ \\
\hline$\sigma(\theta)$ & 7440 & 6700 & 5300 & 3200 & 1440 & 544 & 301 & 226 & 217 & 204 & 19700 \\
\hline
\end{tabular}

the time that $N_{i}$ incident beam ions, corrected for neutralization, were collected. The quantity $(l \Delta \Omega)_{\text {eff }}$ was calculated using standard geometrical equations [27].

In this configuration, the collision chamber and beam line pressures were sufficiently high that corrections had to be made to the data for the loss of ion beam current by charge neutralization along the beam path. The greatest such correction was at $20 \mathrm{keV}$, where the capture cross section for $\mathrm{H}^{+}+\mathrm{H}_{2}$ was taken to be $6.4 \times 10^{-16} \mathrm{~cm}^{2}$ [24]. With a beam line pressure of $10^{-5}$ Torr, approximately $16 \%$ of the protons are neutralized between the analyzing

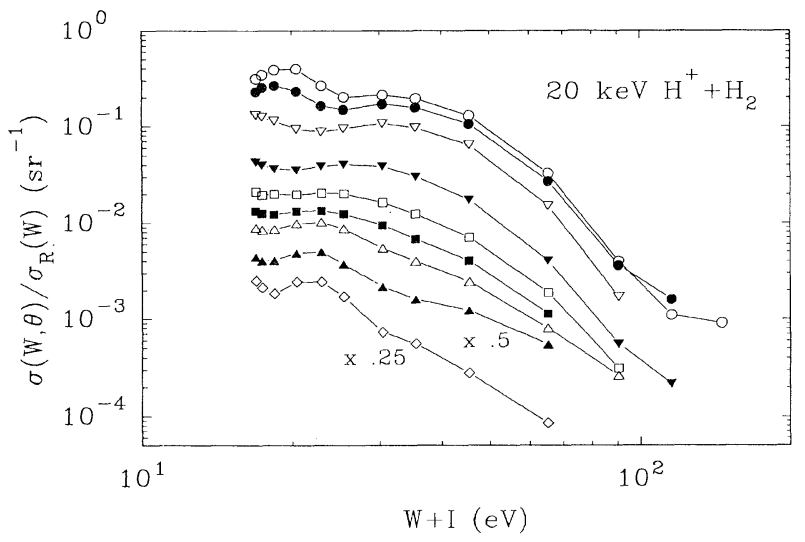

FIG. 1. DDCSs for secondary-electron production in 20-keV $\mathbf{H}^{+}+\mathbf{H}_{2}$ collisions. Cross sections divided by the Rutherford SDCSs are plotted vs $W+I$, where $W$ is the secondary energy and $I$ the ionization potential $(15.43 \mathrm{eV})$. The angles for the curves are, from top to bottom, $15^{\circ}, 20^{\circ}, 30^{\circ}, 50^{\circ}, 70^{\circ}, 90^{\circ}, 110^{\circ}$, $130^{\circ}$, and $160^{\circ}$. The last two curves have been multiplied by 0.5 and 0.25 to avoid overlap. magnet and the Faraday cup, the fraction decreasing for higher energies. For the $\mathrm{He}^{+}+\mathrm{He}$ measurements, the corresponding neutralization fraction at the lowest energy $\mathrm{He}^{+}$used was $4.6 \%$. Since there are no measured DDCS data for neutral $\mathrm{H}$ or $\mathrm{He}$ impact on $\mathrm{H}_{2}$, no further correction could be made for the difference in the ionization of the target by the neutral component of the projectile beam and that due to ions. (This is tantamount to assuming that the DDCS for neutral impact is the same as that for ion impact and correcting only for the ion current not measured.)

The fraction of electrons lost by scattering from the residual gas along their trajectories between the interaction volume and the detector was calculated using an analyti-

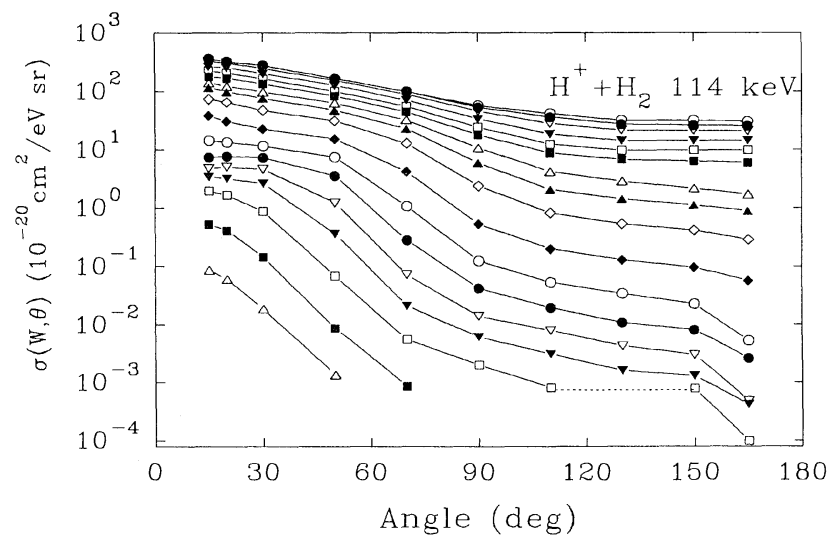

FIG. 2. Angular distributions of DDCSs from 114-keV $\mathbf{H}^{+}+\mathbf{H}_{2}$. The electron energies for the curves are, from top to bottom, 1.5, 2, 3, 5, 7.5, 10, 15, 20, 30, 50, 75, 100, 130, 160, 200, 250 , and $300 \mathrm{eV}$. 


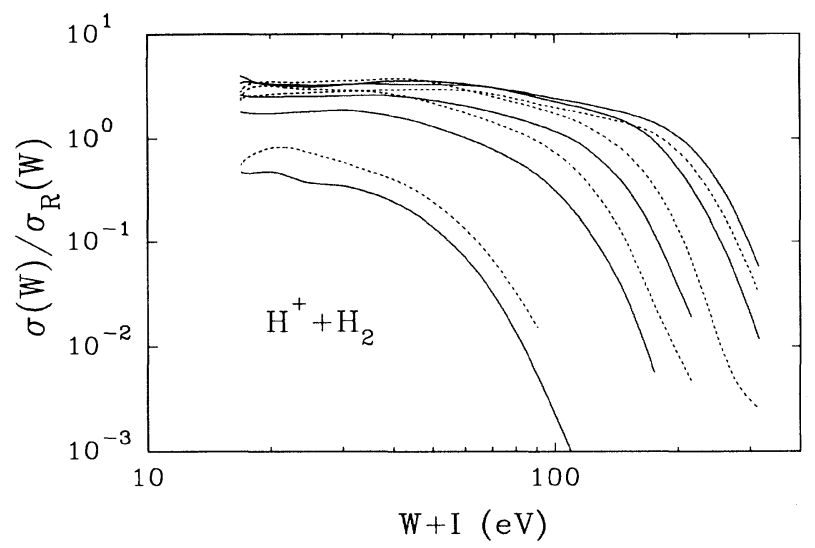

FIG. 3. SDCSs for electrons from $\mathrm{H}^{+}+\mathrm{H}_{2}$ collisions. Solid lines, present data at energies (from lower left to upper right) of $20,48,67,95$, and $114 \mathrm{keV}$; dotted lines, data of Rudd [9] at energies of $20,50,70$, and $100 \mathrm{keV}$.

cal fit to the total electron scattering cross section data of Golden, Bandel, and Salerno [28]. The largest value of this correction was $8 \%$ at an electron energy of $2 \mathrm{eV}$.

Absolute cross sections with the static gas were measured at each combination of ion energy and angle to normalize the relative cross sections. The shapes of the energy spectra for the absolute and relative measurements were very similar except at the very lowest energies and at the extreme backward angles where there were some differences at the highest energies. The $10-\mathrm{eV}$ electron energy chosen for the normalization was in a region where the shapes agreed well.

\section{RELIABILITY OF THE DATA}

\section{A. Molecular targets}

The uncertainties in the relative cross sections are due mostly to the effect of stray fields on the electron trajectories. These added to uncertainties in the analyzer potentials and in the beam collection yield a combined uncertainty of $8 \%$ for $W \geq 10 \mathrm{eV}$, increasing to $17 \%$ at 1.5 eV.

The estimated uncertainties in the quantities in Eq. (3), which yield the absolute DDCSs at $10 \mathrm{eV}$ to normalize the relative data, are $4 \%$ in $\Delta W, 6 \%$ in $\epsilon, 8 \%$ in $n, 5 \%$ in

TABLE VI. Measured values of $\sigma_{i}$ for $\mathrm{H}^{+}+\mathrm{H}_{2}$ in units of $10^{-16} \mathrm{~cm}^{2}$.

\begin{tabular}{ccc}
\hline \hline$E_{p}(\mathrm{keV})$ & $\sigma_{i}$ (expt.) & $\sigma_{i}(\mathrm{rec} .)^{\mathrm{a}}$ \\
\hline 20 & 1.03 & 1.18 \\
48 & 2.14 & 2.08 \\
67 & 2.39 & 2.15 \\
95 & 2.41 & 1.99 \\
114 & 1.97 & 1.86 \\
\hline \hline
\end{tabular}

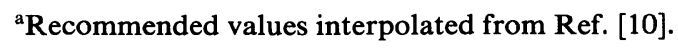

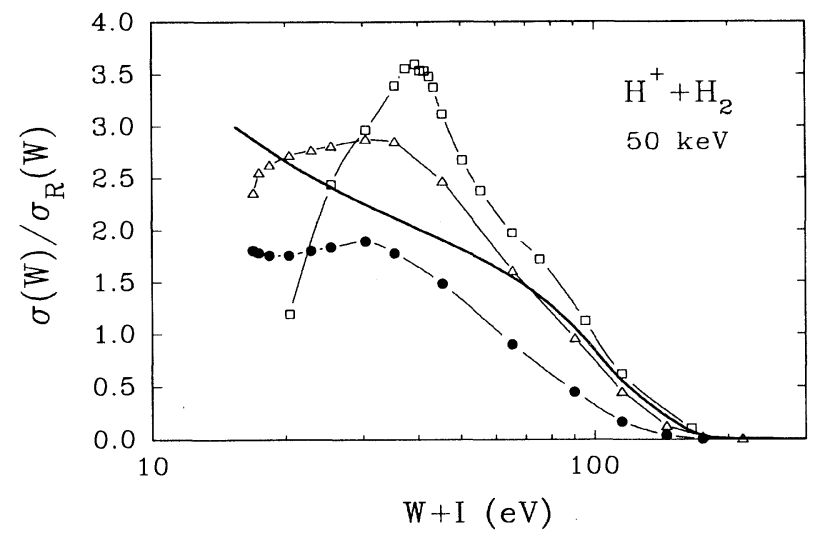

FIG. 4. Energy distributions of electrons from $\mathbf{H}^{+}+\mathrm{H}_{2}$ collisions. Singly differential cross sections divided by the Rutherford cross section are plotted vs $W+I$. Filled circles, present data at $48 \mathrm{keV}$; triangles, data of Rudd [9] at $50 \mathrm{keV}$; squares, data of Gibson and Reid [5] at $50 \mathrm{keV}$; heavy solid line, planewave Born approximation [4] at $50 \mathrm{keV}$.

$N_{i}, 2 \%$ in $N_{e}$, and $10 \%$ in $(l \Delta \Omega)_{\text {eff }}$. Adding these in quadrature yields a total uncertainty of $16 \%$. The absolute cross sections then have a combined uncertainty of $18 \%$ for $W \geq 10 \mathrm{eV}$, increasing to $21 \%$ at $1.5 \mathrm{eV}$. At the highest electron energies where the cross sections are very small and the count rates low the uncertainty increased to about $50 \%$.

\section{B. Atomic targets}

The uncertainty in $\sigma_{1}(W, \theta)$ is the combination of the uncertainties in $R_{12}$ and $\sigma_{2}(W, \theta) . R_{12}$, given by Eq. (2), depends on three signals, rf power off, rf power on, and

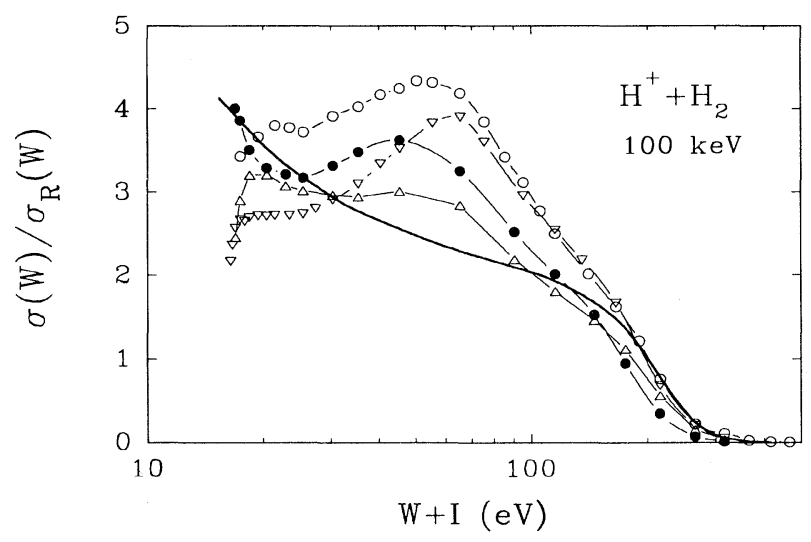

FIG. 5. SDCSs for electrons from $\mathbf{H}^{+}+\mathrm{H}_{2}$ collisions. Filled circles, present data at $95 \mathrm{keV}$; open circles, data of Rudd, Sautter, and Bailey [8] at $100 \mathrm{keV}$; inverted triangles, data of Rudd and Jorgensen [7] at $100 \mathrm{keV}$; triangles, data of Rudd [9] at $100 \mathrm{keV}$; heavy solid line, plane-wave Born approximation [4] at $100 \mathrm{keV}$. 
background, as well as the dissociation fraction $D$. Since the uncertainty in $D$ is only $2 \%$, counting statistics dominate the uncertainty in $R_{12}$, which was typically $12 \%$. The final uncertainties in the atomic cross sections are $22 \%$ above $10 \mathrm{eV}$, increasing to $26 \%$ at $1.5 \mathrm{eV}$ and to $50 \%$ or more at the very highest energies.

\section{RESULTS}

Absolute cross sections for electron ejection from $\mathrm{H}_{2}$ by proton impact are presented in Tables I-V. The energy distributions at each angle are given for $20 \mathrm{keV}$ in Fig. 1 and the angular distributions at each secondary electron energy are shown in Fig. 2 for $114 \mathrm{keV}$. Since the cross sections range over four or five orders of magnitude, this wide range is decreased in some of the figures by dividing by the singly differential Rutherford cross section:

$$
\sigma_{R}(W)=\frac{4 \pi a_{0}^{2} R^{2}}{T(W+I)^{2}},
$$

where $a_{0}$ is the Bohr radius, $R$ the rydberg energy, $I$ the ionization potential, and $T=\frac{1}{2} m_{e} v_{p}^{2}$ with $m_{e}$ the electron mass, and $v_{p}$ is the projectile velocity.

A broad peak centered at $W \simeq 6 \mathrm{eV}$ is evident in the backward directions for the 20-keV spectra in Fig. 1. At higher incident energies this peak is less pronounced, but still appears at the same place in the spectrum. It is not present for atomic hydrogen targets (see paper II [13]). Spectra taken at higher resolution failed to resolve any sharp lines. We suggest that the peak is due to a band of autoionization (AI) electrons from highly excited states of $\mathrm{H}_{2}$. The peak disappears as the angle is reduced from $160^{\circ}$ to $70^{\circ}$ probably because the larger continuum cross section at smaller angles masks the AI spectrum. However, a similar peak appears again in the extreme forward directions, but centered at a slightly lower energy. This may be due to a different cause or it might indicate a forward-backward alignment effect in the emission of AI electrons.

The SDCSs are shown in Fig. 3 compared to previous measurements from this laboratory [9] using an earlier apparatus. The differences at the high-energy end of the spectra are mostly due to the somewhat different incident energies. At the lowest electron energies, where the earlier data tend to drop off, the present data are believed to be more reliable because of better suppression of stray fields and spurious electrons. This is also shown in the TICSs (see Table VI), which have a standard deviation of only $12 \%$ from the recommended values [10] based on more accurate direct measurements. This should be compared to the $44 \%$ deviation for the $20-100-\mathrm{keV}$ data in the earlier measurement [9].

In Figs. 4 and 5 further comparisons of the SDCSs are made with previous measurements and with plane-wave Born approximation (PWBA) calculations using the equation of Kuyatt and Jorgensen [4] scaled for molecular hydrogen [8]. A peak in the experimental spectra where the electron and projectile velocities are equal is due to electron capture to the continuum (ECC) [29]. The PWBA does not account for this mechanism and so does not show this peak. The spectrum from Gibson and Reid [5] has a pronounced ECC peak since their measurements extend to $0^{\circ}$, where the ECC contribution is greatest. All of the earlier measurements shown in Figs. 4 and 5 have a dropoff at low energies, while the trend of the present data is upward near $W=0$, in agreement with the Born approximation.

\section{ACKNOWLEDGMENT}

This material is based on work supported by the $\mathrm{Na}$ tional Science Foundation under Grants Nos. PHY9020529 and PHY9119818.
[1] M. E. Rudd and J. H. Macek, Case Stud. At. Phys. 3, 47 (1972).

[2] T. W. Shyn, Phys. Rev. A 45, 2951 (1992).

[3] Erich Blauth, Z. Phys. 147, 228 (1957).

[4] C. E. Kuyatt and T. Jorgensen, Jr., Phys. Rev. 130, 1444 (1963).

[5] D. K. Gibson and I. D. Reid, Radiat. Res. 112, 418 (1987).

[6] Wen-Qin Cheng, M. E. Rudd, and Ying-Yuan Hsu, Phys. Rev. A 40, 3599 (1989).

[7] M. E. Rudd and T. Jorgensen, Jr., Phys. Rev. 131, 666 (1963).

[8] M. E. Rudd, C. A. Sautter, and C. L. Bailey, Phys. Rev. 51, 20 (1966).

[9] M. E. Rudd, Phys. Rev. A 20, 787 (1979).

[10] M. E. Rudd, Y.-K. Kim, D. H. Madison, and J. W. Gallagher, Rev. Mod. Phys. 57, 965 (1985).

[11] M. B. Shah and H. B. Gilbody, J. Phys. B 15, 3441 (1982).

[12] L. H. Toburen and W. E. Wilson, Phys. Rev. A 5, 247 (1972).

[13] G. W. Kerby III, M. W. Gealy, Y.-Y. Hsu, and M. E.
Rudd, following paper, Phys. Rev. A 51, 2256 (1995).

[14] J. Slevin and W. Stirling, Rev. Sci. Instrum. 52, 1780 (1981).

[15] Leisk Engineering (currently VSW Technology, Ltd.), Albert Drive, Burgess Hill, West Sussex, RH15 9NX, England.

[16] J. C. Houver, J. Fayeton, and M. Barat, J. Phys. B 7, 1358 (1974).

[17] D. R. Schultz, R. E. Olson, C. O. Reinhold, M. W. Gealy, George W. Kerby III, Ying-Yuan Hsu, and M. E. Rudd, J. Phys. B 24, L599 (1991).

[18] J. William McGowan, Rev. Sci. Instrum. 38, 285 (1967).

[19] M. A. Bolorizadeh and M. E. Rudd, Phys. Rev. A 33, 882 (1986); M. A. Bolorizadeh, Ph.D. thesis, University of Nebraska, 1984.

[20] Edward A. Kurz, Am. Lab. 11, 67 (1979).

[21] R. M. Wood, A. K. Edwards, and M. F. Steuer, Phys. Rev. A 15, 1433 (1977).

[22] V. V. Afrosimov, G. A. Leiko, Yu. A. Mamaev, and M. N. Panov, Zh. Eksp. Teor. Fiz. 56, 1204 (1969) [Sov. Phys. 
JETP 29, 649 (1969)].

[23] J. B. Crooks, Ph.D. dissertation, University of Nebraska, 1974.

[24] P. M. Stier and C. F. Barnett, Phys. Rev. 103, 896 (1956).

[25] G. W. McClure, Phys. Rev. 148, 47 (1966).

[26] R. T. Brackman and Wade L. Fite, Phys. Rev. 112, 1157 (1958).
[27] C. E. Kuyatt, in Methods of Experimental Physics, edited by L. Marton (Academic, New York, 1968), Vol. 7A, p. 13.

[28] D. E. Golden, H. W. Bandel, and J. A. Salerno, Phys. Rev. 146, 40 (1966).

[29] J. Macek, Phys. Rev. A 140, 235 (1970). 